\title{
Glaucoma: The Neuro-ophthalmologic Differential Diagnosis
}

\author{
${ }^{1}$ Mark L Moster, Matthew D Kay \\ ${ }^{1}$ Division of Neuro-ophthalmology, Albert Einstein Medical Center \\ Department of Neuro-ophthalmology, Wills Eye Institute \\ Department of Neurology, Thomas Jefferson University School of Medicine, Philadelphia, PA
}

\section{INTRODUCTION}

A common challenge in ophthalmology and source of neuroophthalmology consultation is whether to consider an alternative cause for visual loss in a patient with glaucoma. This review will attempt to define the reason for the uncertainties in the diagnosis of glaucoma, review the differential diagnosis from a neuro-ophthalmologic standpoint and provide guidelines for who should be evaluated and what tests should be performed.

\section{Definition of the Problem}

Why must we consider neuro-ophthalmologic disease in patients with glaucoma? Well, the three clinical features we use to identify glaucoma patients, namely visual field defects, cupping of the optic disks and elevation of intraocular pressure may be seen in patients with neuro-ophthalmologic disorders as well. Additionally, glaucomas are such common illnesses that it does not preclude someone from having a second abnormality affecting the optic nerve.

In terms of intraocular pressure, glaucoma patients may have a wide-range of intraocular pressure from the classically considered normal range to extremely high levels. This of course may occur in patients with neurologic disease as well. In addition, patients with ocular hypertension may coincidentally have an optic neuropathy other than glaucoma.

Glaucoma usually presents with characteristic visual field defects. However, neuro-ophthalmologic disease may produce similar field defects. Often there are defects that are less suggestive of glaucoma and more characteristic of an alternative optic neuropathy. One must keep in mind characteristic defects of these other optic neuropathies such as inferior altitudinal defects in ischemic optic neuropathy or temporal field defects in patients with chiasmal lesions.
In our experience, the most confusing issue which leads to the misdiagnosis of glaucoma in neuro-ophthalmologic patients is the lack of realization in the ophthalmic community that cupping may occur in neuro-ophthalmologic disease. In fact, in a series of patients with non-glaucomatous optic atrophy $20 \%$ had cupping and in $6 \%$ this was typical for glaucoma. ${ }^{1}$ When looked at more carefully, it turns out that in addition to cupping, the rim of the optic disk is most often pale in neurologic disease. ${ }^{2}$ Rarely does the cupping extend to completely obliterate the rim in neurologic disease. Non-glaucomatous cupping has been described to varying degrees in patients with compressive lesions of the visual pathway, hereditary optic neuropathies, ischemic optic neuropathy or CRAO, optic neuritis, toxic optic neuropathy, radiation optic neuropathy and neurodegenerative diseases. ${ }^{1}$ Although the cupping in most neurologic diseases may look different than typical glaucomatous cupping, it is our experience that in the ophthalmologic community these are more than occasionally confused.

\section{DISTINGUISHING GLAUCOMA FROM NONGLAUCOMA}

In which patient should we consider a neuro-ophthalmologic problem? Our approach would be to look for atypical features of the history, examination, optic disk appearance or visual field.

A history that favors a neurologic cause is an abrupt visual loss or rapid progression of the visual loss. This would rarely occur in open-angle glaucoma patients. Accompanying ocular motility defects or other neurologic symptoms or signs should raise a red flag. Although, migraine may occur with increased frequency in NTG, persistent headache or ocular pain should also trigger some concern.

On the visual examination, one expects a relatively good visual acuity compared to the degree of cupping in glaucoma 
patients. If the visual acuity, color vision or visual field defect seems out of proportion to the cupping, then neurologic disease must be considered. When examining the visual field, arcuate defects may occur with glaucoma and other optic neuropathies. However, certain field defects are distinctly less common in glaucoma and more common in neurologic disease. These include central or cecocentral visual loss, defects that respect vertical meridian particularly temporal hemianopic defects with chiasmal lesions, and inferior altitudinal defects which are characteristic of AION. Additionally, if the degree of temporal loss seems greater than nasal loss one should consider chiasmal disease.

When evaluating the optic disk, one of the most important points is to assess the degree of pallor, not in the cup but in the rim surrounding the cup. If the preserved rim appears pale, one must consider non-glaucomatous alternatives. Focal or diffuse obliteration of the neural rim, however, is much more typical of glaucoma. In one study, pallor of the neural rim was found to be $94 \%$ specific for non-glaucomatous processes, while focal or diffuse rim obliteration was $87 \%$ specific for glaucoma. Thinning of the neural rim was only $47 \%$ specific for glaucoma. ${ }^{1}$

Additionally, cupping often occurs coincidental to or even long after the visual loss in patients with neurologic disease in contrast to glaucoma where cupping precedes the visual loss.

Additional features that should raise some concern include young age, severe dyschromatopsia or prominent relative afferent pupillary defect (RAPD). An RAPD is infrequently encountered in glaucoma via standard clinical assessment with the swinging flashlight test. However, if there is asymmetric cupping in excess of 0.2 to 0.3 , one may certainly encounter an APD. A study evaluating the incidence of an APD in the setting of asymmetric glaucoma found the mean difference in C:D for the APD group was 0.43 (range 0.2 to 0.6 ) while the mean difference in C:D in those patients with asymmetric glaucoma without an APD was 0.24 (range 0.2 to 0.3 ). ${ }^{3}$ Accordingly, the finding of an APD in a patient with presumed glaucoma with asymmetric cupping of less than 0.3 should certainly raise concern for an underlying non-glaucomatous optic neuropathy.

We will now review many of the neuro-ophthalmologic entities, which may mimic glaucoma.

\section{INFLAMMATORY OPTIC NEUROPATHY}

Optic neuritis may occasionally mimic glaucoma by virtue of the similar visual field defects and cupping of the optic disk that may occur in these conditions. A typical patient with optic neuritis is a young adult, more commonly a woman, with sudden visual loss and pain on eye movement. There is a variable decrease in visual acuity, prominent dyschromatopsia, afferent pupillary defect, and acutely either a normal or swollen optic disk. Eventually, there is optic pallor but there may be some degree of disk cupping in a small percentage of patients. ${ }^{1}$ The difficulty in distinguishing this from glaucoma is when the patient is seen after the acute event if there is cupping of the optic disk and a residual visual field defect. However, the accompanying disk pallor and possible residual dyschromatopsia or decreased central acuity in the setting of post-inflammatory optic atrophy should be helpful clinical features that serve to distinguish this entity from glaucoma.

Other causes of inflammatory optic neuropathy that have been described to mimic glaucoma include sarcoidosis (Figs $1 \mathrm{~A}$ to $\mathrm{C}$ ) and syphilis that have eventually developed cupping.

\section{ISCHEMIC DISEASE}

Ischemic optic neuropathy may be confused with glaucoma when the patient is seen in a non-acute phase. With giant cell arteritis at least $92 \%$ of patients develop optic disk cupping months after the acute event. ${ }^{4,5}$ In nonarteritic ischemic optic neuropathy (NAION) 2 to $14 \%$ will normally develop cupping., One entity that is often confused with NTG is a unilateral or
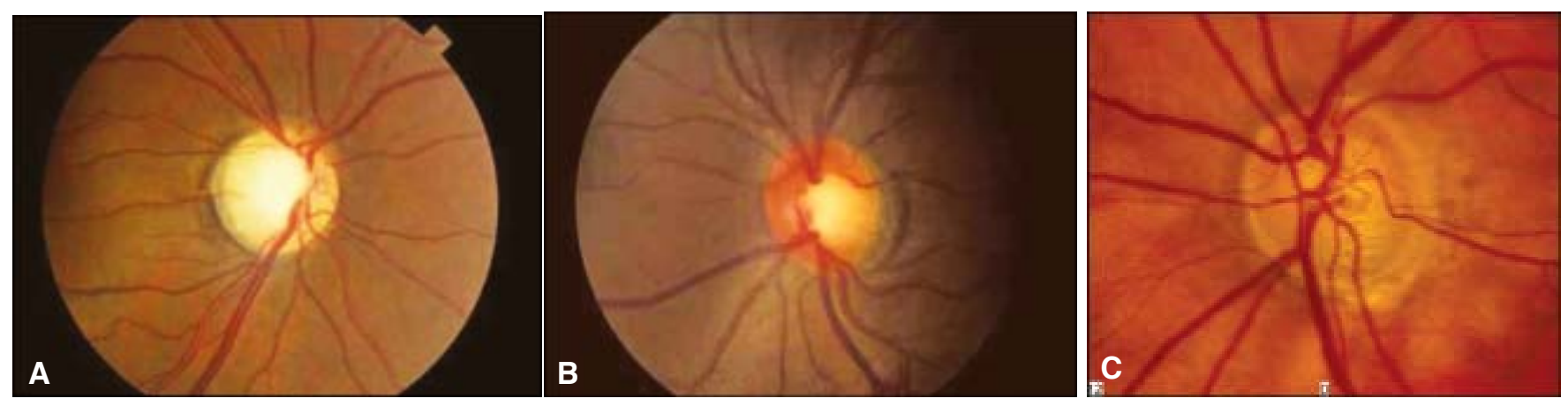

Figs 1A to C: 29 years young man with 20/200 visual acuity OD, 9 months after subacute visual loss. Initial ophthalmologist diagnosed glaucoma but evaluation revealed optic neuropathy from sarcoidosis. Note cupping of the optic disk and pallor of the preserved rim OD (a). OS shows mild cupping and normal color of the optic rim (B). For comparison, (C) shows a more typical glaucomatous optic disk with an inferior notch, peripapillary atrophy and normal color of the optic rim 
bilateral ischemic optic neuropathy from systemic hypotension and/or blood loss. In these patients, the visual field defect will remain stable, as there is no active ongoing glaucomatous process. Additionally, disk pallor will develop weeks to months after the ischemic event. This is a rare complication of surgeries, most commonly cardiac or spinal procedures. ${ }^{6}$

The typical presentation of anterior ischemic optic neuropathy is acute onset of painless optic nerve dysfunction. The visual field loss is typically arcuate or altitudinal in nature, but any type of nerve fiber bundle defect is possible. There is an afferent pupillary defect when the process is unilateral or asymmetric and the optic disk is always swollen acutely.

Occasionally after a central retinal artery occlusion there may be optic disk cupping and so glaucoma may be misdiagnosed. In our experience the cupping is not focal but a shallow diffuse excavation of the optic disk.

Radiation optic neuropathy likely is due to vaso-occlusive disease with optic nerve ischemia. Patients may have visual field defects and disk cupping which can mimic glaucoma. The onset is usually months to years after radiation and the course of visual loss is rapid.

\section{COMPRESSIVE OPTIC NEUROPATHY}

This is the most concerning type of patient who may be misdiagnosed as glaucoma because intervention may be sightsaving and prevent neurologic disability or even death. The problem here is that compressive lesions cause a slowly progressive visual defect that may mimic glaucoma and cupping may occur. In one series of 250 patients with structural visual pathway lesions, 16 had cupping and 5 were misdiagnosed initially as glaucoma. ${ }^{7}$ When looked at carefully these patients really had pallor of the optic disk rim. Compressive lesions of the optic apparatus that may lead to acquired optic nerve cupping include mainly pituitary adenomas, meningiomas, craniopharyngiomas, and aneurysms.

In another study of cupping in compressive optic nerve lesions there was a larger cup-to-disk ratio on the side of the compression (0.37) than in age-matched controls (0.10). Additionally, in those with unilateral compression, the cup-todisk was greater by an average of 0.13 compared to the other side, demonstrating that this cupping was acquired. Control patients had and intereye difference of only 0.04 .

A study comparing the clinical characteristics, visual fields, and optic nerve head appearance of patients with normal tension glaucoma (NTG) and those with intracranial mass lesions affecting the afferent visual pathways found that the presence of a disk hemorrhage was $100 \%$ specific for the diagnosis of NTG. Accordingly, the finding of a peripapillary hemorrhage in a patient with presumed NTG might obviate the need to obtain a neuro-imaging study and other laboratory investigations. Other features with high specificity for the diagnosis of NTG include a family history of glaucoma (96\%), nerve fiber bundle defects (84\%), loss of vertical neuroretinal rim (77\%), and visual field defects bordering the horizontal meridian (77\%). Clinical characteristics which were highly specific for a compressive lesion included age less than 50 years (93\%), optic nerve pallor in excess of cupping (90\%), visual field defects obeying the vertical meridian (81\%), unilateral field defects (80\%), and visual acuity $<20 / 40(77 \%){ }^{8}$

In contrast to other studies, a report of 62 patients with newly diagnosed typical NTG had neuroimaging and 4 patients (6.5\%) had compressive lesions. There were 2 pituitary tumors, 1 meningioma and 1 arachnoid cyst. ${ }^{9}$

Compressive optic neuropathy is the most important alternative entity to consider in glaucoma patients, and we would suggest at least thinking about it as a possibility when initially evaluating a glaucoma patient.

\section{TRAUMATIC OPTIC NEUROPATHY}

Optic neuropathy may occur after severe head injury and rarely after blunt trauma to the brow. Patients have typical findings of optic neuropathy often with severe visual loss. A shallow diffuse cupping is occasionally seen. ${ }^{1}$

\section{HEREDITARY OPTIC NEUROPATHIES}

Most hereditary optic neuropathies, which are slowly progressive, such as autosomal dominant or autosomal recessive optic neuropathy develop pallor of the optic disk without cupping. ${ }^{10}$ However, there are certainly patients with hereditary optic neuropathy who develop cupping. The clues that differentiate these patients from glaucoma are that the rim is pale, the onset is early, the visual loss may be central and there is a family history. ${ }^{11}$

Leber's hereditary optic neuropathy (LHON) presents with acute to subacute visual loss in one eye, followed usually within months by visual loss in the other eye. It is most common in young adult men and is transmitted maternally because it is due to a mitochondrial mutation. It is easily identified by the suddenness and severity of the visual loss along with the typical "pseudo-edema” of the optic disk. However, if not seen acutely, eventually cupping occurs in many patients with Leber's disease. In one study a C:D ratio of 0.7-0.9 was found after the visual loss. ${ }^{12}$ In fact on HRT the findings were quite similar to NTG patients in $73 \%$ of cases. ${ }^{13}$ On the other hand, a study looking at 54 NTG patients failed to identify the major LHON mutations in any of the subjects. ${ }^{14}$ Again, the clinical history should readily permit one to distinguish LHON patients from individuals with glaucoma.

\section{CONGENITAL ENTITIES WHICH MAY MIMIC GLAUCOMA}

Patients with congenital conditions, which include physiologic cupping, myopia, optic pits and coloboma, may have an optic 
disk appearance that mimics glaucoma. The clue here is that there is no significant visual acuity or field loss or a baseline visual loss, which with rare exception, ${ }^{15}$ does not progress overtime. Additionally, with physiologic cupping often the horizontal cup becomes greater than the vertical which is in contrast to glaucoma. Patients with high myopia may be associated with enlarged blind spots in the visual field associated with peripapillary atrophy. Patients with periventricular leukomalacia may have optic disk cupping as a manifestation of trans-synaptic degeneration. ${ }^{16}$

\section{TOXIC OPTIC NEUROPATHY}

Most chronic toxic optic neuropathies develop pallor without cupping. However, in methanol intoxications eventually cupping may occur. ${ }^{17}$ By history, these patients have had acute visual loss.

\section{NEURO-DEGENERATIVE DISEASE}

There has been a literature suggesting that glaucoma may be more common in Alzheimer's disease. ${ }^{18}$ However, our sense is that the degeneration seen in the optic nerve is more likely a function of neuronal degeneration in Alzheimer's disease than a secondary disease such as NTG. The average cup-to-disk ratio in a group of Alzheimer's patients was 0.501 compared to 0.397 in age-matched controls. ${ }^{19}$ Indeed, there has been histologic evidence of neuronal loss in the optic nerve in Alzheimer's disease. ${ }^{20}$ In a Danish study of 11,721 patients with POAG or NTG, no increased risk of Alzheimer's disease was found. ${ }^{21}$

\section{RETRO-CHIASMAL DISEASE}

Lesions behind the optic chiasm are associated with homonymous hemianopsia. If the lesion is anterior to the lateral geniculate nucleus, there may be optic atrophy, sometimes accompanied by cupping. Such lesions may be ischemic, compressive, degenerative or inflammatory. With lesions behind the lateral geniculate in children, there may be trans-synaptic degeneration with optic atrophy and cupping.

\section{INTERMITTENT IOP ELEVATION}

Some patients with apparent NTG have intermittent elevation of IOP not noted on examination. Examples include musicians who play wind instruments, weight lifters, people who stand on their head, use gravity inversion boots, who rub their eyes aggressively or have popping eyes. ${ }^{22-26}$

\section{DIAGNOSTIC EVALUATION}

Now that we have reviewed the differential diagnosis, we must reconsider whom to workup and what workup to perform.
Patients with the features reviewed in the Table 1 deserve consideration for further evaluation. The type of workup should be guided by the individual case, based on the history, exam and visual field defects.

Table 1: Features suggesting a non-glaucomatous diagnosis

\begin{tabular}{ll}
\hline History & Young age \\
\hline & $\begin{array}{l}\text { Rapid onset } \\
\text { Rapid progression } \\
\text { Headache (other than typical migraine) } \\
\text { Other neurologic symptoms }\end{array}$ \\
& $\begin{array}{l}\text { Loss of visual acuity or visual field out of proportion } \\
\text { to cupping } \\
\text { Severe dyschromatopsia }\end{array}$ \\
& $\begin{array}{l}\text { Afferent pupillary defect without significant } \\
\text { asymmetry of cupping } \\
\text { Ocular motility or other neurologic defects }\end{array}$ \\
Visual field & $\begin{array}{l}\text { Atypical visual field: temporal > nasal, respect of } \\
\text { vertical meridian, inferior altitudinal defect, central } \\
\text { scotoma }\end{array}$ \\
& Pallor of preserved rim \\
\hline
\end{tabular}

The most important part of the workup is neuroimaging of the visual pathway. The best test to perform is an MRI of the brain and orbits with high-resolution, fat saturated T1 images through the orbits. The MRI is performed with and without contrast and if for some reason an MRI cannot be performed, a high resolution CT scan with and without contrast can be performed instead. If there is a contraindication to contrast then the studies have to be performed without contrast, which decreases the diagnostic sensitivity.

In neuro-ophthalmology, we often see patients who come in with reports of normal MRI or CAT scans, but who really do have compressive or infiltrative optic nerve lesions. Many factors contribute to this situation. First, the requisition order for the study must be accurate and detail exactly what must be evaluated. Secondly, there must be appropriate expertise in interpreting the films, preferably by a neuro-radiologist. Optimally one knows the neuro-radiologist interpreting the films just as one would know the retina specialist that you might refer a patient because what we really are doing when we order an MRI or CAT scan is that we are requesting a neuro-radiologic consultation.

Although some authors have recommended performing neuroimaging in typical cases of NTG, ${ }^{9}$ we would reserve the imaging for atypical cases.

In terms of serologic testing, we recommend testing for any of the known underlying causes of optic neuropathy. Therefore, 
we would check for B12 level, folate, RPR, FTA, Lyme titer, ANA, ESR and angiotensin-converting enzyme (ACE) level. If Leber's hereditary optic neuropathy is a consideration then send mitochondrial DNA studies looking for the Leber's mutations. If sarcoidosis is a possibility, also do chest imaging, beginning with a standard chest X-ray.

Finally, if there is progressive visual loss which does not fit with glaucoma and the neuroimaging and serologic testing are unrevealing, we occasionally proceed to spinal fluid examination to rule out a meningeal inflammatory process such as fungal infection, tuberculosis or lymphomatous or carcinomatous meningitis.

There are a group of patients who are not typical of glaucoma with progressive visual loss who after thorough neuroophthalmologic testing do not yet have a diagnosis. At least one can be comfortable that we are not missing a treatable neurologic cause for their progressive visual loss, and if they otherwise fit like glaucoma to some degree it may be reasonable to continue glaucoma treatment.

A corollary is the possible role of lowering intraocular pressure in individuals with ocular hypertension who coincidentally have an underlying non-glaucomatous optic neuropathy. As it may be difficult to assess for the development or progression of glaucomatous field defects in the setting of an underlying non-glaucomatous optic neuropathy, prophylactic topical therapy to lower IOP in an ocular hypertensive with a co-existing non-glaucomatous optic neuropathy may also be reasonable.

In summary, patients with glaucoma must periodically be re-evaluated to ascertain that the clinical course fits with one of the types of glaucoma. If not, one must consider alternatives and evaluate them appropriately. Evaluation may include neuroophthalmologic consultation, brain and orbital imaging, serologic testing, etc. Additionally, glaucoma is such a common disease that one must not be complacent into thinking that all visual loss is related to that disease.

If the course becomes too rapid or the visual defect becomes atypical, one must reconsider that another process is occurring, the most important being a compressive lesion of the visual pathway.

\section{REFERENCES}

1. Trobe JD, et al. Nonglaucomatous excavation of the optic disk. Arch Ophthalmol 1980;98(6):1046-50.

2. Trobe JD, Glaser JS, Cassady JC. Optic atrophy. Differential diagnosis by fundus observation alone. Arch Ophthalmol 1980;98(6):1040-5.

3. Brown RH, et al. The afferent pupillary defect in asymmetric glaucoma. Arch Ophthalmol 1987;105(11):1540-3.
4. Hayreh SS. Pathogenesis of cupping of the optic disc. Br J Ophthalmol 1974;58(10):863-76.

5. Danesh-Meyer HV, Savino PJ, Sergott RC. The prevalence of cupping in end-stage arteritic and nonarteritic anterior ischemic optic neuropathy. Ophthalmology 2001;108(3):593-8.

6. Rupp-Montpetit K, Moody ML. Visual loss as a complication of non-ophthalmic surgery: a review of the literature. Insight 2005;30(1):10-7.

7. Kupersmith MJ, Krohn D. Cupping of the optic disc with compressive lesions of the anterior visual pathway. Ann Ophthalmol 1984. 16(10):948-53.

8. Greenfield DS, et al. The cupped disc. Who needs neuroimaging? Ophthalmology 1998;105(10):1866-74.

9. Ahmed II, et al. Neuroradiologic screening in normal-pressure glaucoma: study results and literature review. J Glaucoma 2002;11(4):279-86.

10. Kline LB, Glaser JS. Dominant optic atrophy. The clinical profile. Arch Ophthalmol 1979;97(9):1680-6.

11. Fournier AV, et al. Disc excavation in dominant optic atrophy: differentiation from normal tension glaucoma. Ophthalmology 2001;108(9):1595-602.

12. Ortiz RG, et al. Optic disk cupping and electrocardiographic abnormalities in an American pedigree with Leber's hereditary optic neuropathy. Am J Ophthalmol 1992;113(5):561-6.

13. Mashima Y, et al. Optic disc excavation in the atrophic stage of Leber's hereditary optic neuropathy: comparison with normal tension glaucoma. Graefes Arch Clin Exp Ophthalmol 241(2):7580.

14. Opial D, et al. Leber's hereditary optic neuropathy mitochondrial DNA mutations in normal-tension glaucoma. Graefes Arch Clin Exp Ophthalmol 2001;239(6):437-40.

15. Moore MD, Salles, Jampol LM. Progressive optic nerve cupping and neural rim decrease in a patient with bilateral autosomal dominant optic nerve colobomas. Am J Ophthalmol 2000;129(4):517-20.

16. Jacobson LA, Hellstrom, Flodmark O. Large cups in normalsized optic discs: a variant of optic nerve hypoplasia in children with periventricular leukomalacia. Arch Ophthalmol 1997;115(10):1263-9.

17. Stelmach MZ, O’Day J. Partly reversible visual failure with methanol toxicity. Aust NZJ Ophthalmol 1992;20(1):57-64.

18. Bayer AU, Ferrari F, Erb C. High occurrence rate of glaucoma among patients with Alzheimer's disease. Eur Neurol 2002;47(3):165-8.

19. Tsai CS, et al. Optic nerve head and nerve fiber layer in Alzheimer's disease. Arch Ophthalmol 1991;109(2):199-204.

20. Sadun AA, Bassi CJ. Optic nerve damage in Alzheimer's disease. Ophthalmology 1990;97(1):9-17.

21. Kessing LV, et al. No increased risk of developing Alzheimer disease in patients with glaucoma. J Glaucoma 2007;16(1):4751. 
22. Friberg TR, Weinreb RN. Ocular manifestations of gravity inversion. Jama 1985;253(12):1755-7.

23. Slamovits T. Popping eyes. Surv Ophthalmol 1989;33(4):27380.

24. Pecora L, Sibony P, Fourman S. Eye-rubbing optic neuropathy. Am J Ophthalmol 2002;134(3):460-1.

25. Schuman JS, et al. Increased intraocular pressure and visual field defects in high resistance wind instrument players. Ophthalmology 2000;107(1):127-33.

26. Chromiak JA, et al. Resistance training exercises acutely reduce intraocular pressure in physically active men and women. J Strength Cond Res 2003;17(4):715-20.

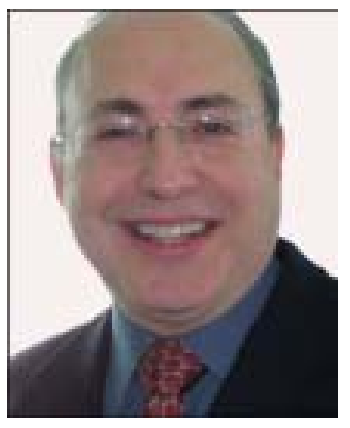

Mark L Moster (markmoster@gmail.com)

\section{Symptoms of Inner Peace}

An unmistakable ability to enjoy each moment.

A tendency to think and act spontaneously, rather than on fears based on past experiences.

A loss of interest in interpreting the actions of others and judging other people.

A loss of interest in conflict.

A loss of ability to worry.

Frequent, overwhelming episodes of appreciation.

Contented feelings of connectedness with others and nature.

Frequent attacks of smiling.

An increased susceptibility to the love extended by others, as well as the uncontrollable urge to extend it.

Inner peace is contagious...

Pass it on ! 